\title{
LOCALIZATION IN NON-NOETHERIAN GROUP RINGS
}

\author{
by P. F. SMITH
}

(Received 26 April, 1979)

1. Introduction. Let $k$ be a field and $G$ an Abelian group of finite torsion-free rank. 3rewer, Costa and Lady [1, Theorem A] showed that if $k$ has characteristic 0 then each scalization of the group algebra $k G$ at a prime ideal is a regular local ring. They also howed (in the same theorem) that if $k$ has characteristic $p>0$, then $k G$ is locally Joetherian (i.e. each localization of $k G$ at a prime ideal is a Noetherian ring) if and only f $G$ is an extension of a finitely generated group by a torsion $p^{\prime}$-group. The purpose of his note is to examine this theorem in a more general setting.

Let $R$ be a ring (with identity) and $P$ a semiprime ideal of $R$. An element $c$ of $R$ is egular if $c r \neq 0$ and $r c \neq 0$ for every non-zero element $r$ of $R$. Let

$$
\mathscr{C}_{R}(P)=\{c \in R: c+P \text { is a regular element of the ring } R / P\} .
$$

Ne shall write $\mathscr{C}(P)$ for $\mathscr{C}_{R}(P)$ when there is no ambiguity about the ring $R$. We shall say hat $P$ is localizable if $R$ satisfies the right and left Ore conditions with respect to $\mathscr{C}(P)$; .e. given $r$ in $R$ and $c$ in $\mathscr{C}(P)$ there exist elements $r_{1}, r_{2}$ in $R$ and $c_{1}, c_{2}$ in $\mathscr{C}(P)$ with

$$
r c_{1}=c r_{1} \quad \text { and } \quad c_{2} r=r_{2} c \text {. }
$$

f $P$ is a localizable semiprime ideal of $R$ let

$$
T(P)=\{r \in R: c r d=0 \text { for some elements } c, d \text { in } \mathscr{C}(P)\} .
$$

Then $T=T(P)$ is an ideal of $R$ and $c+T$ is a regular element of the ring $R / T$ for each slement $c$ in $\mathscr{C}(P)$. Moreover, we can form the partial (right and left) quotient ring of $R / T$ with respect to $\{c+T: c \in \mathscr{C}(P)\}$ and we denote it by $R_{P}$.

Let $k$ be a field and $G$ a group. Let $g$ be the augmentation ideal of the group algebra $k G$. We first consider when $g$ is localizable. This is certainly the case if $G$ is locally iilpotent. For, given any elements $r$ in $k G$ and $c$ in $\mathscr{C}(\mathbf{g})$ there exists a finitely generated iubgroup $H$ such that $r \in k H$ and $c \in \mathscr{C}(\mathfrak{h})$, where $\mathfrak{h}$ is the augmentation ideal of $k H$. But $\mathfrak{b}(\mathfrak{h}) \leqslant \mathscr{C}(\mathfrak{g})$ and it is well known that $\mathfrak{h}$ is localizable. Hence $\mathfrak{g}$ is localizable.

Our first main result is the following one.

THEOREM A. Let $k$ be a field of characteristic $0, G$ a poly-(finitely generated Abelian or locally finite) group and $\mathfrak{g}$ the augmentation ideal of the group algebra $k G$. Then the following statements are equivalent.

(i) $g$ is localizable.

(ii) $\mathrm{g}$ has the AR property.

(iii) $G$ is an extension of a locally finite group by a nilpotent group having each upper central factor of finite torsion-free rank.

Recall that an ideal $I$ of a ring $R$ has the $A R$ property if for any right ideal $E$ and left Glasgow Math. J. 21 (1980) 151-163. 
ideal $L$ there exists a positive integer $n$ such that

$$
E \cap I^{n} \leqslant E I \text { and } L \cap I^{n} \leqslant I L .
$$

For any prime $p$ let $\mathfrak{S}_{\mathrm{p}}$ denote the class of groups $G$ having a finite chain

$$
1=H_{0} \leqslant H_{1} \leqslant \ldots \leqslant H_{n}=G
$$

of normal subgroups $H_{i}$ of $G$ such that $H_{i} / H_{i-1}$ is finitely generated Abelian or locally finite- $p^{\prime}$ for each $1 \leqslant i \leqslant n$. A result for fields of non-zero characteristic corresponding to Theorem $\mathrm{A}$ is the following.

TheOrem B. Let $k$ be a field of characteristic $p>0, G$ an $\mathfrak{S}_{\mathrm{p}}$-group and $\mathrm{g}$ the augmentation ideal of the group algebra $R=k G$. Then the following statements are equivalent.

(i) $\mathfrak{g}$ is localizable.

(ii) $g$ has the $A R$ property.

(iii) $G$ centralizes all p-chief factors.

By a $p$-chief factor of $G$ we mean a chief factor each of whose non-trivial elements has order a power of $p$.

We call a ring $S$ with Jacobson radical $J$ quasi-local provided $S / J$ is a simple Artinian ring. Let $P$ be a localizable prime ideal of a ring $R$ and $T=T(P)$. Then the ideal $P R_{P}=\left\{(x+T)(c+T)^{-1}: x \in P, c \in \mathscr{C}(P)\right\}$ of $R_{P}$ is contained in the Jacobson radical of $R_{P}$ and the ring $R_{P} / P R_{P}$ is isomorphic to the (classical) quotient ring of $R / P$. Thus, by [2, Theorems 4.1 and 4.4], $R_{P}$ is a quasi-local ring provided the ring $R / P$ is a (right and left) Goldie ring; on the other hand if $R_{P}$ is a (right and left) Noetherian ring then so is $R_{P} / P R_{P}$ and hence $R / P$ is a Goldie ring. (Note that all chain conditions will be assumed to hold on both sides unless specified otherwise.) We shall call a semiprime ideal $Q$ of $R$ an annihilator semiprime ideal if $R / Q$ satisfies the ascending chain condition on right annihilators and on left annihilators. Of course, if $R$ is a commutative ring then all prime ideals of $R$ are localizable annihilator prime ideals.

A ring $R$ is called a regular local ring if $R$ is Noetherian quasi-local with Jacobson radical $M$ such that there exists a finite chain

$$
M=M_{0}>M_{1}>\ldots>M_{t}=0
$$

of ideals $M_{i}$ of $R$ such that $M_{i-1} / M_{i}$ is generated by a central regular element of $R / M_{i}$ for each $1 \leqslant i \leqslant t$. In this case, Walker [12, Theorem 2.7] proved that $R$ is prime and $t$ is the global dimension of $R$, the Krull dimension of $R$, the homological dimension of the $R$-module $R / M$ and the supremum of the lengths of chains of prime ideals of $R$, and we call $t$ the dimension of $R$.

If $G$ is a group and $p$ a prime or zero then by $O_{p^{\prime}}(G)$ we mean the intersection of all the normal subgroups $N$ of $G$ for which $G / N$ has no non-trivial finite- $p^{\prime}$ normal subgroup. By a finite- $O^{\prime}$ group we shall mean an arbitrary finite group. Let $\mathfrak{N}_{p}$ denote the class of groups $G$ such that $G / O_{p^{\prime}}(G)$ is a nilpotent group each of whose upper central factors is an extension of a finitely generated group by a torsion $p^{\prime}$-group. For such a group $G$ let 
$h(G)$ denote the sum of the torsion-free ranks of the upper central factors of $G / O_{p^{\prime}}(G)$. It is not hard to prove that $h(G)$ is an invariant for $G$.

THEOREM C. Let $k$ be a field of characteristic $0, G$ an $\Re_{0}$-group and $P$ an annihilator prime ideal of the group algebra $R=k G$. Then $P$ is localizable and $R_{\mathrm{P}}$ is a regular local ring of dimension at most $h(G)$.

The situation for fields of non-zero characteristic is rather different. Firstly we have:

THEOREM D1. Let $k$ be a field of characteristic $p>0, G$ an $\mathfrak{N}_{\mathrm{p}}$-group and $P$ an annihilator prime ideal of the group algebra $R=k G$. Then $P$ is localizable and $R_{\mathrm{P}}$ is a Noetherian ring.

If $p$ is a prime let $\mathfrak{R}_{p}^{*}$ denote the class of $\mathfrak{R}_{\mathrm{p}}$-groups $G$ such that each upper central factor of $G / O_{p^{\prime}}(G)$ is an extension of a free Abelian group of finite rank by a torsion $p^{\prime}$-group. For $\mathfrak{M}_{p}^{*}$-groups we have the following result.

THEOREM D2. Let $k$ be a field of characteristic $p>0, G$ an $\mathfrak{N}_{p}^{*}$-group and $P$ an annihilator prime ideal of the group algebra $R=k G$. Then $R_{\mathrm{P}}$ is a regular local ring of dimension at most $h(G)$.

Note that Theorems C and D2 generalize not only [1, Theorem A] but also [9, Theorem B].

2. Proofs of Theorems $A$ and B. Let $R$ be a ring and $I$ an ideal of $R$. Define a chain of ideals

$$
I=I^{1} \geqslant I^{2} \geqslant \ldots \geqslant I^{\alpha} \geqslant I^{\alpha+1} \geqslant \ldots,
$$

where, for all ordinals $\alpha$,

and

$$
I^{\alpha+1}=I I^{\alpha}+I^{\alpha} I,
$$

$$
I^{\alpha}=\bigcap_{\beta<\alpha} I^{\beta}
$$

if $\alpha$ is a limit ordinal. There exists an ordinal $\rho$ such that $I^{\rho}=I^{\rho+1}$, and for the least such ordinal $\rho$ write

$$
\kappa(I)=I^{\circ} .
$$

Now let $R$ be a Noetherian ring and let $J$ be the Jacobson radical of $R$. Then

$$
\kappa(J)=\kappa(J) J+J \kappa(J)
$$

and since $\kappa(J)$ is finitely generated both as a right ideal and as a left ideal it follows, by Nakayama's Lemma, that

$$
\kappa(J)=0 .
$$

This fact has a simple consequence for localizations of prime ideals. Let $P$ be a localizable prime ideal of $R$ such that $R_{P}$ is a Noetherian ring. If $T=T(P)$ then $P R_{P}=$ $\left\{(p+T)(c+T)^{-1}: p \in P, c \in \mathscr{C}(P)\right\}$ is the Jacobson radical of $R_{\mathrm{P}}$ and so

$$
\kappa\left(P R_{P}\right)=0 .
$$


This gives immediately

Lemma 2.1. Let $P$ be a localizable prime ideal of a ring $R$ such that $R_{\mathrm{P}}$ is a Noetherian ring. Then $\kappa(P) \leqslant T(P)$.

We wish to push this lemma somewhat further. If $P$ is a localizable prime ideal of $R$ we define

and

$$
T_{r}(P)=\{r \in R: r c=0 \text { for some } c \text { in } \mathscr{C}(P)\},
$$

$$
T_{l}(P)=\{r \in R: c r=0 \text { for some } c \text { in } \mathscr{C}(P)\} .
$$

Recall the following well-known result.

Lemma 2.2. Let $R$ be a ring which satisfies the ascending chain condition on right annihilators and let $P$ be a localizable prime ideal of $R$. Then $T(P)=T_{r}(P)$.

Proof. Let $r \in R$ and $c \in \mathscr{C}(P)$ with $c r=0$. If $r(x)$ denotes the right annihilator of the element $x$ of $R$ then

$$
r(c) \leqslant r\left(c^{2}\right) \leqslant \ldots
$$

and there exists a positive integer $n$ such that

$$
r\left(c^{n}\right)=r\left(c^{n+1}\right) .
$$

There exist elements $s$ in $R$ and $d$ in $\mathscr{C}(P)$ such that $c^{n} s=r d$. Then $c r=0$ implies $r d=0$. It follows that $T(P)=T_{r}(P)$.

A non-empty subset $S$ of a ring $R$ will be called an Ore set if

(i) $S$ is multiplicatively closed,

(ii) for all elements $r$ of $R$ and $t$ of $S$ there exist elements $r_{1}, r_{2}$ of $R$ and $t_{1}, t_{2}$ of $S$ such that $r_{1}=t r_{1}$ and $t_{2} r=r_{2} t$, and

(iii) $\{r \in R: r t=0$ for some $t$ in $S\}=\{r \in R: t r=0$ for some $t$ in $S\}$.

In this case let $T(S)=\{r \in R: r t=0$ for some $t$ in $S\}$. The partial quotient ring of $R$ with respect to $S$ will be denoted by $R_{S}$.

Lemma 2.3. Let $P$ be a localizable prime ideal of a ring $R$ such that there exists an Ore set $S$ with $S \leqslant \mathscr{C}(P)$ and $R_{S}$ Noetherian. Then $\kappa(P) \leqslant T_{r}(P)$.

Proof. By Lemmas 2.1 and 2.2,

$$
\kappa\left(P R_{\mathrm{S}}\right) \leqslant T_{\mathrm{r}}\left(P R_{\mathrm{S}}\right)
$$

where $P R_{S}=\left\{(p+T(S))(t+T(S))^{-1}: p \in P, t \in S\right\}$. If $r \in \kappa(P)$ then there exist $c$ in $\mathscr{C}(P)$ and $t$ in $S$ such that $r c t=0$. Since $t \in \mathscr{C}(P)$ it follows that $r \in T_{r}(P)$. Hence $\kappa(P) \leqslant T_{r}(P)$.

Let $k$ be a field and $G$ a group. Then the augmentation ideal of the group algebra $k G$ will be denoted by $g_{k}$ or simply $g$ when there is no ambiguity about $k$.

Lemma 2.4. Let $k$ be a field and $G$ a group such that $g_{k}$ is localizable. If $H$ is any subgroup of $G$ then $\mathfrak{h}_{k}$ is localizable. 
Proof. Let $r \in k H$ and $c \in \mathscr{C}(\mathfrak{h})$. Then $c \in \mathscr{C}(\mathrm{g})$ and there exist elements $s, d$ in $k G$ with $d$ in $\mathscr{C}(\mathbf{g})$ such that $r d=c s$. Let $T$ be a transversal to the right cosets of $H$ in $G$. Then $k G=\underset{t \in T}{\oplus}(k H) t$. It follows that there exist elements $s^{\prime}, d^{\prime}$ in $k H$ with $d^{\prime}$ in $\mathscr{C}(\mathfrak{h})$ such that $r d^{\prime}=c s^{\prime}$. It follows that $\mathfrak{h}$ is localizable.

Proof of Theorem A. The equivalence of (ii) and (iii) is proved in [11, Theorem D]. Also (ii) implies (i) by [10, Lemma 2.2]. Thus it is sufficient to prove that (i) implies (iii). Let

$$
1=H_{0} \leqslant H_{1} \leqslant \ldots \leqslant H_{\mathrm{n}}=G
$$

be a finite chain of subgroups $H_{i}$ of $G$ such that $H_{i-1}$ is normal in $H_{i}$ and $H_{i} / H_{i-1}$ is finitely generated Abelian or locally finite for each $1 \leqslant i \leqslant n$. We prove the result by induction on $n$. If $n=1$ then $\mathrm{g}$ has the AR property by [10, Theorem C]. So suppose $n>1$ and let $H=H_{n-1}$. By Lemma 2.4 we can suppose that $\mathfrak{h}$ has the AR property and $G / H$ is either finitely generated Abelian or locally finite.

Suppose that $G / H$ is finitely generated Abelian. Since $\mathfrak{h}$ has the AR property it follows that $S=\{1-a: a \in \mathfrak{h}\}$ is an Ore set in $k H$ and the ring $(k H)_{S}$ is Noetherian (see [10, Lemma 2.2 and Corollary C1]). Because $S$ is $G$-invariant, $S$ is an Ore set in $R$, where $R=k G$ (see the proof of [6, Lemma 13.3.5 (ii)]), and by [6, Theorem 10.2.6] $R_{\mathrm{S}}$ is a Noetherian ring. Since $S \leqslant \mathscr{C}(\mathrm{g})$ we can apply Lemma 2.3 to obtain

$$
\kappa(\mathbf{g}) \leqslant T_{r}(\mathbf{g})
$$

On the other hand, suppose that $G / H$ is locally finite. By [11, proof of Theorem E], for every finitely generated right ideal $E$ of $R$ there exists a positive integer $m$ such that

$$
E \cap \mathfrak{g}^{m} \leqslant E \mathfrak{g}
$$

and by [10, Lemma 2.1] we conclude

$$
\mathfrak{g}^{\omega}=\bigcap_{m=1}^{\infty} \mathfrak{g}^{m}=T_{r}(\mathfrak{g}) .
$$

Thus, in any case,

$$
\kappa(\mathfrak{g}) \leqslant T_{r}(\mathfrak{g}) .
$$

Returning to the chain (1) we note that $G$ has a finite series

$$
1=K_{0} \leqslant K_{1} \leqslant \ldots \leqslant K_{\mathrm{q}}=G
$$

of subgroups $K_{i}$ such that $K_{i-1}$ is normal in $K_{i}$ and $K_{i} / K_{i-1}$ is infinite cyclic or locally finite for $1 \leqslant i \leqslant q$. If $G=G_{1} \geqslant G_{2} \geqslant \ldots$ is the lower central series of $G$ then, arguing as in the proof of [11, Theorem D], $G_{q+1} / G_{q+2}$ is a torsion group. It follows that if $U=G_{q+1}$ then

$$
\mathfrak{u} \leqslant \mathfrak{g}^{\omega} \text {. }
$$

Suppose that $\boldsymbol{u} \leqslant \mathfrak{g}^{\alpha}$ for some ordinal $\alpha$. If $u \in U$ and $x \in G$ then

$$
1-[u, x]=u^{-1} x^{-1}\{(1-x)(1-u)-(1-u)(1-x)\} \in \mathrm{g}^{\alpha+1} \text {. }
$$


Since $G_{q+1} / G_{q+2}$ is a torsion group it follows that $u \leqslant g^{\alpha+1}$. Thus $\mathfrak{u} \leqslant \kappa(g) \leqslant T_{r}(g)$. Now it is easy to prove that $U$ is a locally finite group (see the proof of [11, Theorem D]). This proves (iii).

Theorem A has the following consequence.

COROLlary A. Let $k$ be a field of characteristic 0 and $G$ a hyper-(Abelian or locally finite) group and let $\mathrm{g}$ be the augmentation ideal of the group algebra $R=k G$. Then the following statements are equivalent.

(i) $\mathfrak{g}$ is localizable, $T_{1}(\mathfrak{g})=T_{r}(\mathrm{~g})$ and $R_{\mathrm{g}}$ is a Noetherian ring.

(ii) $g$ has the $A R$ property.

(iii) $G$ is an extension of a locally finite group by a nilpotent group with each upper central factor of finite torsion-free rank.

To prove Corollary A, by the theorem we need show only that (i) and (ii) are equivalent. By [10, Lemmas 2.1 and 2.2 and Corollary C1], (ii) implies (i). In order to prove that (i) implies (ii) we require some notation.

Let $P$ be a localizable prime ideal of a ring $R$. If $E$ is a right ideal of $R$ then the $P$-closure of $E$ is

$$
\operatorname{cl}_{P} E=\{r \in R: r c \in E \text { for some } c \text { in } \mathscr{C}(P)\} .
$$

Then $\mathrm{cl}_{P} E$ is a right ideal containing $E$. We call $E P$-closed provided $E=\mathrm{cl}_{\mathrm{P}} E$. There are similar definitions for left ideals. The next lemma is elementary.

Lemma 2.5. Let $P$ be a localizable prime ideal of a ring $R$ such that $T(P)=T_{r}(P)$. Then the ring $R_{\mathrm{P}}$ is right Noetherian if and only if $R$ satisfies the ascending chain condition on $P$-closed right ideals.

To complete the proof of Corollary A, suppose that (i) holds. By Lemma 2.5, $R=k G$ satisfies the ascending chain condition on $g$-closed right ideals. By [11, Lemma $B$ and the proof of Lemma A], $G$ is poly-(locally finite or finitely generated Abelian) and so (iii) follows by Theorem A. This completes the proof of Corollary A.

We now turn our attention to Theorem $B$.

Proof of Theorem B. (ii) and (iii) are equivalent by [11, Theorem E]. Moreover, (ii) implies (i) by [10, Lemma 2.2]. It remains to prove that (i) implies (iii).

Suppose that (i) holds. In order to prove (iii) it is sufficient to prove that if $A$ is a minimal normal subgroup of $G$ and a $p$-group then $A$ is central. There exists a chain

$$
A=H_{0} \leqslant H_{1} \leqslant \ldots \leqslant H_{n}=G
$$

of normal subgroups $H_{i}$ of $G$ such that $H_{i} / H_{i-1}$ is finitely generated Abelian or locally finite- $p^{\prime}$ for each $1 \leqslant i \leqslant n$. The result is proved by induction on $n$. The case $n=0$ is clear since $A$ is finite. So suppose $n>0$ and let $H=H_{n-1}$. By induction we can suppose that $\mathfrak{h}$ has the AR property in $k H$. Then following the argument used in the proof of Theorem A we obtain

$$
\kappa(\mathfrak{g}) \leqslant T_{r}(\mathfrak{g})
$$


If $A$ is not central then $A=[A, G]$ and it follows that

$$
\mathfrak{v} \leqslant \kappa(\mathfrak{g})
$$

so that $A$ is a $p^{\prime}$-group, a contradiction. (The argument is very like that in the proof of Theorem $A$ and so the details are left to the reader.)

In the same way that Theorem A gives Corollary A, Theorem B gives the following result. The proof is virtually identical to that of Corollary A and so is omitted.

COROLlary B. Let $k$ be a field of characteristic $p>0, G$ a hyper-(finitely generated Abelian or locally finite- $p^{\prime}$ ) group and $\mathrm{g}$ the augmentation ideal of the group algebra $R=k G$. Then the following statements are equivalent.

(i) $g$ is localizable, $T_{1}(\mathbf{g})=T_{r}(\mathbf{g})$ and $R_{\mathfrak{g}}$ is a Noetherian ring.

(ii) $g$ has the $A R$ property.

(iii) $G$ is an $\mathfrak{S}_{\mathrm{p}}$-group and $G$ centralizes all $p$-chief factors.

Corollaries A and B should be compared with [11, Theorem C], where it is proved that if $k$ is any field, $G$ a locally nilpotent group and $g$ the augmentation ideal of $R=k G$ then statements (i) and (ii) of Corollary B are equivalent. In fact, for any group $G$, (ii) implies (i) (see [10, Lemmas 2.1 and 2.2 and Corollary C1]). This leaves the question of whether (i) always implies (ii).

3. Proofs of Theorems C, D1 and D2. The key result required is an old result of D. G. Higman (see [6, Lemma 7.2.2]). We call a ring $R$ a Higman extension of a ring $S$ if $S$ is a subring of $R$ with the same identity and there exists a finite collection of units $u_{i}$ $(1 \leqslant i \leqslant n)$ in $R$ such that

(i) $n$ is a unit in $R$,

(ii) $u_{i} S=S u_{i}(1 \leqslant i \leqslant n), u_{i} S \neq u_{i} S(1 \leqslant i \neq j \leqslant n)$,

(iii) $\left\{S u_{i} u_{j}: 1 \leqslant j \leqslant n\right\}=\left\{S u_{j}: 1 \leqslant j \leqslant n\right\}(1 \leqslant i \leqslant n)$, and

(iv) $R=u_{1} S+\ldots+u_{n} S$.

Higman's Lemma can be expressed in the following form.

Lemma 3.1. Any Higman extension of a semiprime Artinian ring is semiprime Artinian.

Corollary 3.2. Let $R$ be a Higman extension of a semiprime Goldie ring $S$. Let $I$ be an ideal of $R$ such that $\mathscr{C}_{S}(0) \leqslant \mathscr{C}_{R}(I)$. Then $I c=0$ for some element $c$ of $\mathscr{C}_{R}(I)$.

Proof. By [2, Theorems 4.1 and 4.4], $S$ has a semiprime Artinian quotient ring $Q$. By [6, Lemma 13.3.5], $\mathscr{C}_{S}(0)$ is an Ore set in the ring $R$ and we denote the partial quotient ring of $R$ with respect to $\mathscr{C}_{S}(0)$ by $Q_{1}$. Clearly $Q_{1}$ is a Higman extension of $Q$ and so, by the lemma, $Q_{1}$ is semiprime Artinian. Because $\mathscr{C}_{S}(0) \leqslant \mathscr{C}_{R}(I)$, it follows that $I Q_{1}=$ $\left\{a c^{-1}: a \in I, c \in \mathscr{C}_{S}(0)\right\}$ is an ideal of $Q_{1}$ and so is generated by a central idempotent element $b d^{-1}$ (say) with $b$ in $I$ and $d$ in $\mathscr{C}_{S}(0)$. Then $I(d-b)=0$ and $d-b \in \mathscr{C}_{R}(I)$.

An ideal $I$ of a ring $R$ has a weak centralizing set of generators if there exists a finite chain of ideals

$$
0=I_{0} \leqslant I_{1} \leqslant \ldots \leqslant I_{n}=I
$$


such that, for each $1 \leqslant j \leqslant n, I_{j} / I_{j-1}$ is generated by a finite collection of central elements of $R / I_{j-1}$ or is $\mathscr{C}(I)$-torsion (i.e. for all $a$ in $I_{j}$ there exist $c_{1}$ and $c_{2}$ in $\mathscr{C}(I)$ such that $a c_{1} \in I_{j-1}$ and $\left.c_{2} a \in I_{j-1}\right)$. If each of the factors $I_{j} / I_{j-1}(1 \leqslant j \leqslant n)$ is generated by a finite collection of central elements of $R / I_{j-1}$ then we say that $I$ has a centralizing set of generators.

We extend these definitions in the following way. Let $R$ be a ring and $G$ a group of automorphisms of $R$. If $r \in R$ and $g \in G$ then

$$
r^{g}
$$

will denote the action of $g$ on $r$. An element $c$ of $R$ will be called $G$-central if $c$ is central in $R$ and

$$
c^{\mathrm{g}}=\mathrm{c}
$$

for all $g$ in $G$. Then $G$-invariant ideals having a weak $G$-centralizing set of generators or a $G$-centralizing set of generators will have the obvious meaning.

We say that an ideal $I$ of a ring $R$ has the right fAR property if for every finitely generated right ideal $E$ there exists a positive integer $n$ such that $E \cap I^{n} \leqslant E I$. The ideal $I$ will be said to have the right $f A R$ property locally if for every finitely generated right ideal $E$ there exists a positive integer $n$ such that

$$
E \cap I^{n} \leqslant \mathrm{cl}_{I}(E I)
$$

i.e. for each element $r$ in $E \cap I^{n}$ there exists $c$ in $\mathscr{C}(I)$ such that $r c \in E I$.

Suppose that $I$ is an ideal of $R$ such that $I$ has the right fAR property locally. Let $E$ be a finitely generated right ideal of $R$ and suppose

$$
x \in \bigcap_{n=1}^{\infty} \mathrm{cl}_{I}\left(E+I^{n}\right) .
$$

If $F=E+x R$ then there exists a positive integer $m$ such that

$$
F \cap I^{m} \leqslant \mathrm{cl}_{I}(F I) \text {. }
$$

There exist $c$ in $\mathscr{C}(I)$ and $e$ in $E$ such that $x c-e \in F \cap I^{m}$ and so $(x c-e) d \in F I \leqslant E+x I$ for some element $d$ of $\mathscr{C}(I)$. It follows that $x \in \mathrm{cl}_{I} E$. Hence

$$
\bigcap_{n=1}^{\infty} \mathrm{cl}_{I}\left(E+I^{n}\right)=\mathrm{cl}_{I} E
$$

for all finitely generated right ideals $E$ of $R$. We require this fact in the proof of the next result.

Lemma 3.3. Let $Q$ be a localizable annihilator semiprime ideal of a ring $R$ such that $Q$ has a weak centralizing set of generators and $Q$ has the right fAR property locally. Then $R_{Q}$ is a right Noetherian ring.

Proof. Let $Y$ be a right ideal of $R_{Q}$ and $Y_{1}=\{r \in R: r+T \in Y\}$, where $T=T(Q)$. Then $Y_{1}$ is a $Q$-closed right ideal of $R$. Moreover, $Y$ is a finitely generated right ideal of $R_{Q}$ if and only if there exists a finitely generated right ideal $Y_{2}$ of $R$ such that $Y_{1}=\operatorname{cl}_{Q} Y_{2}$. 
Suppose there exists a $Q$-closed right ideal of $R$ which is not the $Q$-closure of a finitely generated right ideal. By Zorn's Lemma there exists a $Q$-closed right ideal $E$ maximal with respect to not being the $Q$-closure of a finitely generated right ideal. Suppose that $Q \leqslant E$. Since $Q$ has a weak centralizing set of generators it follows that $Q R_{Q}$ is a finitely generated right ideal of the ring $R_{Q}$. But the ring $R_{Q} / Q R_{Q}$ is isomorphic to the classical right quotient ring $B$ of the ring $R / Q$ and, by [4, Theorem], $B$ is semiprime Artinian. It follows that $(E / T) R_{Q}$ is a finitely generated right ideal of $R_{Q}$. This implies that $E$ is the $Q$-closure of a finitely generated right ideal of $R$, a contradiction.

Thus $Q \neq E$. Because $Q$ has a weak centralizing set of generators there exists a finitely generated right ideal $X_{1}$, an ideal $X$ and an element $c$ of $Q$ such that $\operatorname{cl}_{O} X_{1}=X \leqslant$ $E, c$ is central modulo $X$ and $c \notin E$. Let $F=\{r \in R: c r \in E\}$. Then $F$ is a $Q$-closed right ideal of $R$ and $E \leqslant F$. Let $G=E+c R$. The choice of $E$ entails that there exist a positive integer $n$ and elements $g_{i}(1 \leqslant i \leqslant n)$ of $G$ such that

For each $1 \leqslant i \leqslant n$ let

$$
G \leqslant \mathrm{cl}_{\mathrm{Q}}\left(g_{1} R+\ldots+\mathrm{g}_{n} R\right) .
$$

$$
g_{i}=e_{i}+c r_{i}
$$

with $e_{i}$ in $E$ and $r_{i}$ in $R$. Let $H=e_{1} R+\ldots+e_{n} R$.

Suppose $E \neq F$. Then by the choice of $E$ there exists a finitely generated right ideal $M$ such that $F=\operatorname{cl}_{\mathrm{Q}} M$. Let $e \in E$. Then $e \in G$ and hence there exists an element $d$ in $\mathscr{C}(Q)$ such that

$$
e d=\sum_{i=1}^{n} e_{i} s_{i}+c u
$$

for some elements $s_{i}(1 \leqslant i \leqslant n)$ and $u$ in $R$. It follows that $u \in F$ and hence $e \in$ $\mathrm{cl}_{\mathrm{Q}}(H+c M)$. But this implies that $E=\mathrm{cl}_{Q}(H+c M)$ and, because $H+c M$ is a finitely generated right ideal, we have a contradiction. Thus $E=F$. In this case $E \leqslant \mathrm{cl}_{Q}(H+c E)$. Using the fact that $c$ is central modulo $X=\mathrm{cl}_{\mathrm{Q}} X_{1}$, it follows that

$$
E \leqslant \bigcap_{s=1}^{\infty} \mathrm{cl}_{Q}\left(H+X_{1}+c^{s} E\right) \leqslant \bigcap_{s=1}^{\infty} \mathrm{cl}_{Q}\left(H+X_{1}+Q^{s}\right)=\mathrm{cl}_{Q}\left(H+X_{1}\right),
$$

by (2). Hence $E=\mathrm{cl}_{\mathrm{Q}}\left(H+X_{1}\right)$, another contradiction. The result follows.

LEMMA-3.4. Let $k$ be a field of characteristic $p \geqslant 0$ and $G$ an $\mathfrak{R}_{\mathrm{p}}$-group. Let $P$ be an annihilator prime ideal of the group algebra $R=k G$. Then $P$ is localizable, $P$ has a weak centralizing set of generators and $R_{P}$ is a Noetherian ring.

Proof. There exists an infinite chain

$$
1=H_{0} \leqslant H_{1} \leqslant \ldots \leqslant H_{\alpha} \leqslant H_{\alpha+1} \leqslant \ldots \leqslant H_{\rho}=G
$$

of normal subgroups $H_{\alpha}$ of $G$ such that for all ordinals $\alpha$,

(i) $H_{\alpha+1} / H_{\alpha}$ is an infinite cyclic group or a finite- $p$ group and $\left[H_{\alpha+1}, G\right] \leqslant H_{\alpha}$, or and

(ii) $H_{\alpha+1} / H_{\alpha}$ is a finite- $p^{\prime}$ group,

$$
H_{\alpha}=\bigcup_{\beta<\alpha} H_{\beta}
$$


if $\alpha$ is a limit ordinal. Moreover, all but a finite number of the factors $H_{\alpha+1} / H_{\alpha}$ are finite- $p^{\prime}$ groups. For each ordinal $\alpha$ with $0 \leqslant \alpha \leqslant \rho$ let $R^{(\alpha)}=k H_{\alpha}$ and $P^{(\alpha)}=P \cap k H_{\alpha}$. Then $P^{(\alpha)}$ is a $G$-invariant annihilator semiprime ideal of $R^{(\alpha)}$ for each ordinal $\alpha$ with $0 \leqslant \alpha \leqslant \rho$. To see that $R^{(\alpha)} / P^{(\alpha)}$ satisfies the ascending chain condition on right annihilators one need merely note that for any non-empty subset $X$ of $R^{(\alpha)}$,

$$
R^{(\alpha)} \cap\{r \in R: X r \leqslant P\}=\left\{r \in R^{(\alpha)}: X r \leqslant P^{(\alpha)}\right\} .
$$

If $N$ is the ideal of $R^{(\alpha)}$ containing $P^{(\alpha)}$ such that $N / P^{(\alpha)}$ is the sum of all nilpotent ideals of $R^{(\alpha)} / P^{(\alpha)}$ then $N$ is $G$-invariant and, by [3, Theorem 1], $N / P^{(\alpha)}$ is nilpotent. It follows that $N R$ is an ideal of $R$ and $(N R)^{s} \leqslant P$ for some positive integer $s$. Hence $N R \leqslant P$ and it follows that $P^{(\alpha)}$ is semiprime.

Next we claim that, for each ordinal $\alpha$ with $0 \leqslant \alpha \leqslant \rho$,

$$
\begin{aligned}
& P^{(\alpha)} \text { is a localizable ideal of } R^{(\alpha)} \text { such that } P^{(\alpha)} \text { has a weak } \\
& G \text {-centralizing set of generators and } R_{P^{(\alpha)}}^{(\alpha)} \text { is a Noetherian ring. }
\end{aligned}
$$

The action of $G$ on the ring $R^{(\alpha)}$ is by conjugation.

Suppose that (3) is false and let $\alpha$ be the least ordinal for which it fails to be true. Clearly $\alpha>0$. Suppose first that $\alpha$ is not a limit ordinal. Let $A=H_{\alpha-1}, B=H_{\alpha}$, $P_{1}=P^{(\alpha-1)}, P_{2}=P^{(\alpha)}, S=R^{(\alpha-1)}$ and $T=R^{(\alpha)}$. Then $P_{1}=P_{2} \cap S$. By hypothesis, $P_{1}$ is localizable in $S$. Hence $T$ satisfies the right and left Ore conditions with respect to $\mathscr{C}_{S}\left(P_{1}\right)$ (see [6, Lemma 13.3.5]). Let $U=\mathscr{C}_{S}\left(P_{1}\right)$ and

$$
K=\left\{t \in T: t u \in P_{2} \text { for some } u \text { in } U\right\} .
$$

Then $K$ is a $G$-invariant ideal of $T$ and $P_{2} \leqslant K$. By [7, Lemma 7], $P_{2}<K$ implies the existence of an element $t$ of $K$ which is central in $k G$ modulo $P$. But $t u \in P_{2}$ for some $u$ in $U$ and hence $u \in P_{2} \cap S=P_{1}$, a contradiction. Thus $K=P_{2}$ and it follows that

$$
U \leqslant C_{T}\left(P_{2}\right) \text {. }
$$

A similar argument shows that $T_{1}\left(P_{1}\right)=T_{r}\left(P_{1}\right)$.

Now suppose that $B / A$ is a finite- $p^{\prime}$ group. By [4, Theorem], $S / P_{1}$ has a semiprime Artinian quotient ring and, by [2, Theorem 4.4], $S / P_{1}$ is a Goldie ring. Thus we can apply Corollary 3.2 to obtain that $P_{2} / P_{1} T$ is $\mathscr{C}_{T}\left(P_{2}\right)$-torsion. It follows that $P_{2}$ has a weak $G$-centralizing set of generators. By hypothesis $S_{U}$ is a Noetherian ring and hence $T_{U}$, being a finitely generated $S_{U}$-module, is a Noetherian ring. Hence, by [8, Theorem 2.2 Corollary 1], $P_{2} T_{U}$ is localizable and it follows that $P_{2}$ is localizable and $T_{P_{2}}$ is Noetherian.

Next suppose that $B / A$ is infinite cyclic. By [9, Lemma 2.1], either $P_{2}=P_{1} T$ or there exists an element $c$ of $P_{2}$ which is $G$-central and regular modulo $P_{1} T$ (and hence regular modulo $\left.P_{1} R\right)$ such that $P_{2} /\left(P_{1} T+c T\right)$ is $\mathscr{C}_{\mathrm{T}}\left(P_{2}\right)$-torsion. As before, $P_{2}$ has a weak $G$-centralizing set of generators, $P_{2}$ is localizable and $T_{P_{2}}$ is a Noetherian ring.

The other possibility is that $B / A$ is a finite- $p$ group. Then $P_{2} / P_{1} T$ has a $G$ centralizing set of generators (see [7, Lemma 7]) and again $P_{2}$ has the desired properties. Thus $\alpha$ is a limit ordinal. 
Let $\beta<\alpha$. Since $P^{(\beta)}$ is localizable it follows that $R^{(\alpha)}$ satisfies the right and left Ore conditions with respect to

$$
\mathscr{C}_{R^{(\beta)}\left(P^{(\beta)}\right)}
$$

(see [6, Lemma 13.3.5]) and as above

$$
\mathscr{C}_{\mathbf{R}^{(\beta)}}\left(P^{(\beta)}\right) \leqslant \mathscr{C}_{\mathbf{R}^{(\alpha)}}\left(P^{(\alpha)}\right) .
$$

It follows that

$$
\mathscr{C}_{R^{(\alpha)}}\left(P^{(\alpha)}\right)=\bigcup_{0 \leqslant \beta<\alpha} \mathscr{C}_{R^{(\beta)}\left(P^{(\beta)}\right)}
$$

Consequently, $P^{(\alpha)}$ is localizable.

Since only a finite number of the factors $H_{\beta+1} / H_{\beta}$ are not finite- $p^{\prime}$ groups, there exists an ordinal $\gamma$ with $0 \leqslant \gamma<\alpha$ such that $H_{\beta+1} / H_{\beta}$ is a finite- $p^{\prime}$ group for each ordinal $\beta$ with $\gamma \leqslant \beta<\alpha$. Thus $H_{\alpha} / H_{\gamma}$ is a locally finite- $p^{\prime}$ group and by the argument used earlier in the proof, $P^{(\alpha)} / P^{(\gamma)} R^{(\alpha)}$ is $\mathscr{C}\left(P^{(\alpha)}\right)$-torsion. It follows that $P^{(\alpha)}$ has a weak $G$-centralizing set of generators.

Let $X=R^{(\alpha)}, Y=R^{(\gamma)}$ and $V=\mathscr{C}\left(P^{(\gamma)}\right)$. Since $P^{(\gamma)} Y_{V}$ has a centralizing set of generators it follows that $P^{(\gamma)} Y_{V}$ has the AR property in $Y_{V}$ (see $[5,2.7]$ ). By adapting the proof of [11, Theorem E], we conclude that for each finitely generated right ideal $E$ of $X$ there exists a positive integer $m$ such that for each element $r$ of $E \cap X\left(P^{(\gamma)}\right)^{m}$ there exists an element $c$ of $V$ such that $r c \in E P^{(\gamma)}$. Since $P^{(\alpha)} / X P^{(\gamma)}$ is a right $\mathscr{C}\left(P^{(\alpha)}\right)$-torsion module it follows that $P^{(\alpha)}$ has the right fAR property locally in $X$. Hence by Lemma 3.3, $X_{P^{(\alpha)}}$ is a right Noetherian ring. Similarly it is a left Noetherian ring as well. This contradicts the choice of $\alpha$ and completes the proof of Lemma 3.5.

Theorem D1 follows at once from Lemma 3.4. Now let $k$ be a field of characteristic $p \geqslant 0$ and $G$ an $\mathfrak{N}_{0}$-group (if $p=0$ ) or an $\mathfrak{N}_{\mathrm{p}}^{*}$-group (if $p \neq 0$ ). If $P$ is an annihilator prime ideal of the ring $R=k G$ then by the proof of Lemma 3.5 we see that there exists a finite chain

$$
0=P_{0} \leqslant P_{1} \leqslant \ldots \leqslant P_{n}=P
$$

of ideals $P_{i}$ of $R$ such that $P_{i} / P_{i-1}$ is generated by a central regular element of $R / P_{i-1}$ or $P_{i} / P_{i-1}$ is $\mathscr{C}(P)$-torsion for all $1 \leqslant i \leqslant n$. Moreover, $P$ is localizable and it follows that $R_{\mathrm{P}}$ is a regular local ring. By examining the proof of Lemma 3.5 we see that the dimension of $R_{\mathrm{P}}$ is at most $h(G)$. This completes the proof of Theorems C and D2.

Finally we mention an analogous result for integral group rings. Let $\mathfrak{X}$ denote the class of Abelian groups $G$ which contain a free Abelian subgroup $F$ of finite rank such that $G / F$ is a torsion group with finite $p$-primary component for each prime $p$.

THEOREM 3.5. Let $G$ be a nilpotent group each of whose upper central factors is an $\mathfrak{X}$-group and let $R$ be the integral group ring $Z G$. If $P$ is an annihilator prime ideal of $R$ then $\boldsymbol{P}$ is localizable and $\boldsymbol{R}_{\boldsymbol{P}}$ is a Noetherian ring. 
Proof. If $P \cap Z=0$ then the non-zero elements of $Z$ belong to $\mathscr{C}_{R}(P)$. If $Q$ is the rational field then $P^{\prime}=P Q$ is an annihilator prime ideal of $S=Q G$ and, by Theorem $C$, $R_{P} \cong S_{P^{\prime}}$ is a regular local ring. If $P \cap Z \neq 0$ then there exists a prime $p$ such that $p \in P$. By Theorem D1, $\bar{P}=P / p R$ is localizable and, if $\bar{R}=R / p R, \bar{R}_{\bar{P}}$ is Noetherian. It can easily be checked that $p R$ has the right fAR property and $P / p^{n} R$ is localizable for all integers $n \geqslant 1$. Let $r \in R, c \in \mathscr{C}(P)$. There exists a positive integer $m$ such that

$$
(r R+c R) \cap p^{m} R \leqslant(r R+c R) p .
$$

There exist elements $s$ in $R$ and $d$ in $\mathscr{C}(P)$ such that $r d-c s \in p^{m} R$ and, hence,

$$
r d-c s=(r a+c b) p
$$

for some $a, b$ in $R$. Thus

$$
r(d-a p)=c(s+b p)
$$

and $d-a p \in \mathscr{C}(P)$. It follows that $P$ is localizable. Also $R_{P} / p R_{P}$ is a right Noetherian ring. By adapting the proof of Lemma 3.3, $R_{P}$ is a right Noetherian ring. Similarly $R_{P}$ is a left Noetherian ring.

Finally we can combine Corollaries A and B and Theorems C and D1 to characterize, for hypercentral groups $G$, those group algebras $R=k G$ such that every annihilator prime ideal $P$ is localizable with $R_{P}$ a Noetherian ring. Note that for such a prime ideal $P$, we have, by [7, Theorem A],

$$
T_{1}(P)=T_{r}(P) .
$$

THEOREM 3.6. Let $k$ be a field of characteristic $p \geqslant 0$ and $G$ a hypercentral group. Then a necessary and sufficient condition for every annihilator prime ideal $P$ of the group algebra $R=k G$ to be localizable with $R_{P}$ a Noetherian ring is that $G$ be an $\Re_{p}$-group.

\section{REFERENCES}

1. J. W. Brewer, D. L. Costa and E. L. Lady, Prime ideals and localization in commutative group rings, $J$. Algebra 34 (1975), 300-308.

2. A. W. Goldie, Semiprime rings with maximum condition, Proc. London Math. Soc. (3) 10 (1960), 201-220.

3. I. N. Herstein and L. W. Small, Nil rings satisfying certain chain conditions, Canad. J. Math. 16 (1964), 771-776.

4. R. E. Johnson and L. S. Levy, Regular elements in semiprime rings, Proc. Amer. Math. Soc. 19 (1968), 961-963.

5. Y. Nouazé and P. Gabriel, Idéaux premiers de l'algèbre enveloppante d'une algèbre de Lie nilpotente, J. Algebra 6 (1967), 77-99.

6. D. S. Passman, The algebraic structure of group rings (Wiley-Interscience, 1977).

7. J. E. Roseblade and P. F. Smith, A note on hypercentral group rings, J. London Math. Soc. (2) 13 (1976), 183-190.

8. P. F. Smith, Localization and the AR property, Proc. London Math. Soc. (3) 22 (1971), 39-68. 
9. P. F. Smith, On non-commutative regular local rings, Glasgow Math. J. 17 (1976), 98-102.

10. P. F. Smith, The AR property and chain conditions in group rings, Israel J. Math. 32 (1979), 131-144.

11. P. F. Smith, More on the AR property and chain conditions in group rings, Israel J. Math., to appear.

12. R. Walker, Local rings and normalizing sets of elements, Proc. London Math. Soc. (3) 24 (1972), 27-45.

Department of Mathematics

UNIVERSITY GARDENS

Glasgow G12 8QW 